In Crescendo, 2020; 11(2): 225-238

Fecha de recepción: 21/05/2020

Fecha de aceptación: 03/06/2020

\title{
EDUCACIÓN VIRTUAL INTERACTIVA COMO METODOLOGÍA PARA LA EDUCACIÓN: REVISIÓN DE LITERATURA
}

\author{
INTERACTIVE VIRTUAL EDUCATION AS A METHOD \\ FOR EDUCATION: LITERATURE REVIEW
}

\section{Omar Bullón-Solis}

\section{RESUMEN}

El servicio educativo, en todos sus niveles, migró a la modalidad virtual como medio para continuar operando durante el estado de emergencia sanitaria, producto de la pandemia del COVID 19. Sin embargo, su implementación fue acelerada y en múltiples casos quedó pendiente la formulación y evaluación de las metodologías didácticas adaptadas al nuevo entorno virtualizado. Este trabajo presenta el análisis y la revisión bibliográfica de la implementación de herramientas tecnológicas en el proceso de virtualización de la educación y la respuesta metodológica del docente; para lo cual se empleó el enfoque cualitativo y el método de estudio fenomenológico. El objetivo fue indagar sobre la implementación de las plataformas educativas digitales en las instituciones educativas y la respuesta adaptativa de la comunidad estudiantil. Se encontró que las plataformas educativas son la principal herramienta tecnológica de las instituciones educativas, sin embargo no se realizó la adecuación pedagógica para emplear correctamente estas tecnologías digitales. Se identificó que los docentes requieren dominar el uso de las TIC como herramientas educativas para brindar una enseñanza adecuada a los requerimientos de la sociedad moderna. Se evidenció que la metodología interactiva permite aprovechar las ventajas de las plataformas educativas, por lo que se sugiere como metodología adecuada para la educación virtual.

PALABRAS CLAVE: educación a distancia, metodología interactiva, plataforma educativa.

1 Magíster en Educación. Docente en la Universidad de Ayacucho Federico Froebel.

E-mail: obullon@ucvvirtual.edu.pe ; https://orcid.org/0000-0002-7614-2254 


\begin{abstract}
The educational service migrated to virtual mode to continue operating during the state of health emergency of the COVID 19 pandemic. However, its implementation was accelerated and in multiple cases the developing and evaluation of didactic methodologies was pending to adapted to the new virtualized environment. This work presents the analysis of the bibliographic review of the implementation of technological tools in the process of virtualization and the methodological response of the teacher; for which a qualitative approach and phenomenological study method were used. The but was to investigate the implementation of digital educational platforms in the institutions and the adaptive response of the student community. It was found that educational platforms were the main technological tool for educational institutions, however pedagogical adequacy was not realized properly to use these digital technologies. It was identified that teachers need to master the use of TIC as educational tools to provide adequate teaching to the requirements of a modern society. The interactive methodology allows to take advantage of educational platforms, so it is suggested as a potential methodology for virtual education.
\end{abstract}

KEY WORDS: distance learning, interactive methodology, learning platform.

\title{
INTRODUCCIÓN
}

La práctica de la docencia en el siglo XXI requiere incorporar las nuevas tecnologías educativas disponibles en el quehacer cotidiano de la enseñanza, por lo cual resulta clave vincular los entornos virtuales a las competencias para el uso de las tecnologías de la información y comunicación (TIC) de los docentes. Los tiempos modernos requieren de una nueva generación de docentes que cuenten con el manejo tecnológico necesario para operar las nuevas plataformas educativas que se vienen implementando.

Más allá de la exigencia actual, producto de la pandemia por el virus COVID 19, por incorporar rápidamente la modalidad virtual a todo nivel educativo; la adquisición de competencias tecnológicas por parte del docente y el uso de las herramientas educativas del entorno virtual, constituyen una exigencia permanente para el futuro. En este proceso surge la necesidad de los docentes por formarse continuamente en un proceso de adaptación hacia una educación basada en las tecnologías en línea, como nuevo eje de desarrollo educativo para los estudiantes.

Como parte del progreso educativo en la región latinoamericana, las instituciones educativas han adquirido nuevas herramientas y equipos tecnológicos como parte de la innovación de sus procesos educativos, más aún durante el esta- 
do de emergencia. Esta incorporación de tecnología donde resaltan las plataformas y aulas virtuales llevó a los docentes a incursionar en el proceso de migración hacia la virtualidad -(Tapia-Repetto et al., 2019).

Esta migración fue de menor intensidad en las instituciones públicas con una evidente falta de acompañamiento al cambio de entorno, por lo que un objetivo a futuro es que las propuestas de educación virtual cuenten con un respaldo político y de inversión por parte de los gobiernos. Los estados promueven únicamente el acceso a internet y a brindar los equipos tecnológicos que se conecten a este '(Torres \& Rodríguez, 2019), pero las políticas públicas no son claras en cuanto a la promoción de la educación informatizada y no se insiste en la capacitación docente para el uso de las TIC.

Al respecto, América Latina es una región que posee una dispareja competitividad educativa (Rivera-Laylle et al., 2017). Un estudio revela que existen países que cuentan con un nivel superior al $90 \%$ de sus instituciones educativas equipadas con tecnologías de la información y comunicación (TIC), como Chile y Cuba; países como Perú con el $82 \%$ y Colombia con el $61 \%$, y por otra parte existen países con un bajo desarrollo en la implementación de las TIC, logrando solo el equipamiento del 6\% y el 11\% como el caso de Guatemala y Paraguay respectivamente (Sistema de información sobre tendencias educativas en América Latina-SITEAL, 2014).

Esta situación muestra una necesidad por implementar mejoras en el campo educativo concerniente a la implementación de tecnologías modernas en las instituciones educativas que disminuyan la diferencia con los países más desarrollados; al tiempo que se requiere de una formación adecuada de los docentes para el uso de estas tecnologías.

Para evaluar la calidad educativa, usualmente se emplea los estándares o normas técnicas para la educación; las cuales son políticas aprobadas por un organismo nacional o internacional acreditado para formular normas de estandarización. Estos pueden o no ser de obligatorio cumplimiento, dependiendo de la legislación de cada país (Carmona-Suarez, 2017).

La incorporación de estándares de calidad en la educación tiene por objetivo aclarar las políticas institucionales que deben implementarse para garantizar la calidad educativa. Actualmente en la región de América Latina, diversos países han venido impulsando la reforma educativa a través del licenciamiento de sus instituciones educativas, tanto a nivel básico regular como a nivel superior, 
para lo cual se han desarrollado criterios y estándares para la calidad educativa, sin embargo estos no incorporan criterios claros para la evaluación de la educación virtualizada.

El objetivo de las normas técnicas es la incorporación de los estándares de calidad en las instituciones educativas, con el objetivo de establecer procesos que articulen y resalten el diálogo coherente entre los lineamientos operativos y las estrategias institucionales (Carmona-Suarez, 2017). Por ello, es de interés que estos criterios de calidad se encuentren plenamente definidos para la educación virtual. Estas normas técnicas requieren abordar la modalidad virtual debido a que es un proceso moderno que continuará su desarrollo en el tiempo, y se requiere garantizar la calidad educativa también en los entornos virtualizados.

Las instituciones educativas requieren reconocer que el rol que cumplen las plataformas educativas en el desarrollo de la calidad de la educación no debe ser considerado como decisivo, debido a que el eje central requiere enfocarse en el modo de interacción docente y estudiante, a través de un modelo de trabajo interactivo en un entorno digital que incremente la interacción del docente con su aula de clase. Actualmente las plataformas son la cara visible de las instituciones educativas y la percepción del público estudiantil es la de caracterizar a la educación virtual como una educación fría y falta de motivación.

Promover la mejora de la calidad en la educación virtual consiste en integrar nuevas prácticas metodológicas diseñadas para el entorno digital. El desarrollo de plataformas interactivas permite desarrollar motivación en el estudiante, lo que conlleva a fortalecer sus habilidades de autoaprendizaje, se constituye finalmente en una característica de la educación virtual y conlleva a desarrollar el pensamiento crítico de los estudiantes y una consecuente mejora en su educación. Se afirma entonces que logrando contar con estudiantes bien formados, se promueve el desarrollo general del país.

La educación virtual provee de una competencia básica para el mundo globalizado, se refiere al pensamiento analítico basado en la información disponible que actualmente es amplia; con la cual les permita formular ideas integradas para hacer frente a los desafíos de la globalización (Tünnermann, 2008). Por lo tanto, incorporar buenas metodologías educativas en la educación virtual representa una necesidad tanto actual como para el futuro de la educación.

Teniendo en cuenta que es necesario proporcionar profundidad de entendimiento a esta situación y obtener una adecuada metodología que promueva la 
interacción dual entre estudiante y estudiante, así como estudiante y docente en el proceso del aprendizaje, se requiere conocer cómo debe formarse el docente en la actualidad de tal forma que permita generar un impacto significativo en la enseñanza, generando motivación, cohesión grupal y seguimiento del aprendizaje de sus estudiantes.

Es por ello que generar un entorno virtual motivador que influya positivamente en el desarrollo del aprendizaje juega un rol fundamental para mejorar la educación. Entonces, ¿Cuál es una metodología adaptada para el entorno virtual que conlleva a un aprendizaje motivado por parte del estudiante y permita mejorar la calidad educativa?

El presente artículo plantea que una educación virtual interactiva es una herramienta fundamental para aplicar en la metodología básica de la educación virtual. La actividad educativa precisa del constante diseño, implementación y evaluación de las estrategias de motivación aplicadas en el aula de clase. Esta toma una mayor importancia en la educación virtualizada, donde se requiere afianzar la dinámica profesor-estudiante, debido a la carencia motivacional que se produce por la falta del ambiente físico de intercambio.

Partiendo que la motivación es el motor del aprendizaje significativo, se hace necesario identificar las principales estrategias de motivación que requieren ser implementadas en la modalidad virtual y así contribuir a la mejora continua (Duarte-Herrera et al., 2019). La era moderna con las tecnologías disponibles exige contar con estudiantes mejor preparados para su desarrollo en la sociedad del conocimiento, en la cual nos encontramos en sus inicios.

\section{MÉTODO}

Para el desarrollo del presente artículo se aplicó un enfoque cualitativo empleando el método fenomenológico del estudio de la adecuación de las prácticas pedagógicas a la educación virtualizada. Este tipo de investigación se basa en estudiar los eventos en la forma y cómo aparecen (Mieles-Barrera et al., 2015), en esta ocasión basado en los aportes evidenciados en los trabajos académicos de diferentes autores.

Se aplicó el muestreo no probabilístico por conveniencia, con la elección de materiales bibliográficos relevantes para el presente estudio (Barba et al., 2015). Se identificó los trabajos científicos de veinte autores de artículos publicados en revistas indexadas de SciELO.org y Latindex.org. Se utilizó la técnica de análisis 
documental, con lo cual fue factible interpretar cada material estudiado vinculado al contexto del estudio; para lo cual se diseñó una estructura de categorización (Andréu-Abela, 2001) que permitió alinear los contenidos, identificar las características que luego permitió inferir en la caracterización del progreso de la educación virtual en la región latinoamericana.

\section{ARGUMENTACIÓN}

\section{Plataformas educativas digitales}

En la actualidad las actividades educativas en la modalidad virtual están mediadas por tecnologías educativas. Estas herramientas digitales deben ser pensadas y diseñadas bajo la perspectiva que permitan la difusión de la información que contienen y facilitar el intercambio de conocimientos entre los grupos interconectados. En definitiva, la incursión en el uso de plataformas digitales proporcionó una nueva herramienta para la modalidad presencial y generó la aparición de la modalidad mixta, el aprendizaje b-learning, el cual, sin duda generó múltiples ventajas.

Entre las más destacadas se encuentra que ayuda a los estudiantes a desarrollar la capacidad de investigar en el conjunto de material disponible, seleccionar la información relevante y usar la información para elaborar su propio contenido. Sin embargo, este aprendizaje no ocurre por sí solo, siempre se remarca que no es posible abandonar al estudiante, por el contrario es necesario que el docente considere los factores académicos y tecnológicos disponibles (Montes-Martínez et al., 2019) que influyan de manera relevante en los estudiantes para que estos desarrollen sus competencias digitales.

Migrar al aprendizaje en la modalidad b-learning tiene muchas ventajas, entre las que más destacan tenemos que esta modalidad permite a los estudiantes desarrollar naturalmente la capacidad de buscar, seleccionar y utilizar la información; una habilidad que posteriormente conllevará al pensamiento crítico y a la redacción de investigaciones significativas para el aprendizaje.

Sin embargo, esta habilidad no es aprendida de forma automática; sino que es necesaria la labor del docente como intermediador, es quien debe considerar los factores académicos y tecnológicos para guiar al estudiante. En medio de esta dinámica, las plataformas educativas deben impulsar a los estudiantes a desarrollar sus habilidades digitales y apuntar a su trabajo independiente (MontesMartínez et al., 2019). 
Las plataformas educativas están siendo utilizadas para transmitir contenidos e información; es por eso que los entornos tecnológicos diseñados, sirven de instrumento para generar desarrollo y promoción en los diferentes procesos de la enseñanza y aprendizaje dentro de la educación -(Tapia-Repetto et al., 2019) . En este sentido, los docentes con aptitudes de programación están en la capacidad de poder crear los entornos virtuales, frente a ello, los docentes que no poseen estas habilidades, les resultaría difícil crear los sistemas de aplicación pedagógica virtual.

La necesidad de poseer un conocimiento significativo en programación irá de la mano con el conocimiento conceptual de las diferentes materias y su expansión al entorno virtual, los nuevos procesos de enseñanza serán adecuados a la generación actual de los estudiantes '(Torres \& Rodríguez, 2019). Es así que, en la educación a distancia, el aprendizaje digital, no solo se consolida, no solo se interrumpe, sino que estos impulsos no disminuirán porque las tecnologías siempre alentarán la innovación pedagógica en esta área "'(Badia et al., 2017), donde los cambios tecnológicos globales también han provocado cambio general en el modo de desarrollar la educación y han generado oportunidades acumuladas en el campo de la capacitación en línea (López-Catalán \& Prieto-Jiménez, 2018).

Las plataformas actuales tienden a limitar la calidad del producto educativo. Dado que, los resultados sobre la deserción en los cursos MOOC (90\% en promedio) indican claramente esta situación. El desarrollo de un diseño de modelo de aprendizaje más adecuado, basado en las características de los cursos MOOC (diversidad, heterogeneidad y uso masivo) ha cambiado, lo que mejora la calidad de este producto educativo (abandono de alrededor del 70\%) "'(Badia et al., 2017; Fidalgo-Blanco et al., 2015) y por ello, para mantener la posibilidad de tener un producto final en el proceso, el cual sea propio y real, será la mejor forma de presentar una propuesta educativa virtual.

Este enfoque requiere de la transformación de los usuarios de las plataformas educativas. Tanto docentes, estudiantes y administrativos de las instituciones educativas deben superar la aversión a la tecnología y por el contrario, considerarla como su aliada. El objetivo de la educación virtual es convertir a los usuarios en puntos activos del proceso educativo (Morado et al., 2018).

En ese sentido, las personas que eligen cursos en línea teniendo en cuenta la formación que tiene el docente, la metodología aplicada en la enseñanza, el prestigio institucional, los documentos de respaldo, la duración; son aspectos promo- 
vidos por la metodología. donde, la usabilidad, accesibilidad, adaptabilidad y modularidad son caracteres validados y a la vez compartidos a nivel internacional y tomados en cuenta en la estandarización de calidad (Carmona-Suarez, 2017; Rodríguez Gómez et al., 2018). Pero el uso de las TIC también ha tenido mucho éxito en el ámbito institucional y político, pero a nivel práctico, la mayor parte de las propuestas han surgido y se han mantenido gracias a iniciativa personal de los docentes (Veytia-Bucheli \& Contreras-Cipriano, 2019).

\section{Educación virtual interactiva}

El concepto de innovación educativa fue vinculado, en muchas ocasiones, al empleo y uso de nuevas herramientas tecnológicas; donde las tecnologías de información y comunicación (TIC) fueron consideradas como modernidad en el aula de clase. Sin embargo, la innovación en educación trasciende a la implementación de equipos tecnológicos, que son elementos más o menos sofisticados, cuya finalidad es brindar facilidad y acceso, más no garantiza el aprendizaje.

Es más, la innovación en la educación requiere trascender al uso de este, de las TIC, involucrando el uso de tecnologías únicamente como parte del desarrollo de nuevos hábitos educativos. (Borgobello et al., 2019). Por lo tanto, la innovación debe gestarse a nivel pedagógico e incluirse dentro del modelo educativo vigente de la institución.

Los docentes que desarrollan sus labores educativas dentro de los entornos virtuales de aprendizaje enfrentan desafíos para atender las necesidades de los estudiantes. Actualmente ser docente y trabajar con una plataforma educativa virtual únicamente significa proveer información y recoger las pruebas evaluativas para su calificación. Cuando el contexto actual significa que el docente debe mediar y facilitar la transmisión de conocimiento haciendo uso de la plataforma; pero más importante, a través de diseños metodológicos promover la construcción colectiva del conocimiento(Duarte-Herrera et al., 2019).

Con lo cual se puede afirmar que la educación virtual va más allá del uso de plataformas e incursiona en el campo de la innovación metodológica de la enseñanza. Particularmente se resalta que se requiere planear e implementar estrategias motivacionales para promover aprendizajes significativos (Duarte-Herrera et al., 2019). Sin embargo este concepto no se viene desarrollando en el nivel deseado en las instituciones educativas que han migrado a la educación virtual producto de las necesidades del contexto actual. 
La alternativa al escenario descrito es fomentar el desarrollo de aulas virtuales interactivas que contenga como elemento pivotante la motivación del estudiante para aportar a la sesión de clase. Para lograrlo, es una condición que el docente basado en criterios reflexivos elabore el diseño de metodologías educativas alternativas vinculadas a la reflexión, aporte y resolución de cuestiones cotidianas, haciendo uso de las herramientas tecnológicas disponibles.

Los entornos creativos fomentan el aprendizaje autónomo del estudiante, y esto es concordante con la principal característica de la educación virtualizada, el trabajo independiente. Las herramientas que esta provee al estudiante solo son útiles si este encuentra un objetivo a resolver y la suficiente motivación para hacerlo. Las aulas virtuales interactivas cumplen con los requisitos para ser empleadas en la modalidad virtual, debido que presentan los tres elementos claves para el correcto desarrollo de la educación virtualizada.

Primero, fomenta el trabajo individual grupal pero independiente de la presencia del docente; segundo, porque requiere de abundante información para su aplicación y el medio virtualizado lo provee, y tercero porque requiere del pensamiento creativo por parte de los estudiantes, esto último es concordando con el pensamiento analítico y productor de contenidos, que es la característica principal de la educación virtual.

Es así como alcanzar el máximo de las ventajas de la educación virtual, requiere de garantizar una adaptación de la metodología de enseñanza adaptada a la educación virtualizada, cuyo objetivo planteado es el desarrollo de las aulas virtuales interactivas que fomente la creatividad. Para ello, se requiere de un cambio en las habilidades de estudiantes, docentes y miembros de la comunidad estudiantil, en el que no se adquiere conocimiento por repetición, sino por aplicación; y cuya característica es el aprendizaje colectivo y constructivo (Borgobello et al., 2019).

Por lo tanto, la educación virtual es un progreso cuyo propósito es el cambio en el paradigma educativo, dependiente de un docente y un espacio físico. La educación virtual provee la oportunidad de incursionar en otra forma de educar, donde se priorice la creación de habilidades o capacidades, similar a adquirir habilidades suficientes para integrarnos como generadores de conocimiento (Medina-Gamero, 2019). Estas son las fronteras que plantea la educación virtual en los tiempos venideros. 


\section{Competencias para la educación virtualizada}

La migración a la educación virtual ha significado la introducción de herramientas tecnológicas, las mismas que requieren de una modificación en los planes de estudio e incluso en el currículo. Las competencias requeridas para su uso han sido poco apreciadas debido a que la tendencia fue considerarlas como una herramienta complementaria a la formación tradicional. Sin embargo, es necesario considerar que para la migración a la educación virtualizada se requiere de formar nuevas competencias, tanto en estudiantes como en docentes, los mismo que requiere de una adecuación de los planes de estudio y los currículos.

El principal desafío es para aquellos que persisten en mantener el statu quo educativo con las nuevas herramientas pedagógicas que aporta la educación virtual (Montes-Martínez et al., 2019). Estas novedades tecnológicas exigen la adaptación metodológica de la enseñanza, para poder aprovechar las nuevas ventajas que brinda para la educación, que finalmente servirá para afrontar mejor preparados la educación del país.

Por ende, es necesario que las instituciones educativas brinden la formación docente requerida para aplicar las técnicas de aprendizaje en línea, a fin de proporcionar a los estudiantes la experiencia necesaria para utilizar los recursos digitales diarios y desarrollar en ellos las habilidades digitales e informativas requeridas (Duarte-Herrera et al., 2019; Montes-Martínez et al., 2019), donde se englobe un entorno colaborativo virtual que facilite la colaboración entre estudiantes, y entre docentes y estudiantes. Este tipo de espacio logra involucrar al propio estudiante en el desarrollo de su propia educación como elemento gravitante, con lo cual participa plenamente en los procesos educativos.

Se sugiere que el docente moderno valore las TIC como herramientas educativas cotidianas, debido que estas están vinculadas al progreso educativo y participan de la revolución educativa a nivel mundial, con lo cual están influyendo en el modo de educar a través de la transformación de los procesos educativos y creando la necesidad de interconexión como futuro de la educación. (LópezCatalán \& Prieto-Jiménez, 2018). Sobre ello, un estudio de las TIC a una población de docentes en cierta institución educativa en Guatemala; reflejó que el $57 \%$ de ellos decidieron no participar en el ensayo cuyo objetivo era una evaluación sobre sus conocimientos y sus prácticas tecnológicas en el aula (RiveraLaylle et al., 2017). 
La razón que el estudio argumentó fue que los docentes se sintieron intimidados de participar en la investigación educativa al considerar que este era una crítica a sus métodos pedagógicos utilizados. La realidad actual del docente es que es imperativo que quienes enseñan requieren además de estar capacitados con los conocimientos pertinentes en su materia, también desarrollen habilidades idóneas para transmitir el conocimiento empleando las tecnologías disponibles, así como mantener una actitud de servicio a la formación de sus educandos (RiveraLaylle et al., 2017), con lo cual se gesta la necesidad de capacitar en el uso eficaz de las TIC.

Los programas de capacitación y formación docente apuntan a mejorar las habilidades tecnológicas de los docentes. Para lo cual se requiere identificar las competencias digitales mínimas requeridas para el uso de las tecnologías educativas disponibles, y realizar un estudio de las posibilidades a futuro. Por ello desarrollar propuestas que involucren estrategias tecnológicas y educativas, son más idóneas, porque conlleva a involucrar al componente humano operador de los equipos que se desean adquirir.

En este proceso se considera involucrar a los docentes en el rol de evaluador de las propuestas tecnológicas, y constituyen un incentivo y valoración de la labor del docente (Morado, 2017). Sin obviar la situación que los equipos tecnológicos tienen debilidades y fallas cotidianas dentro de la normalidad, que pueden generar frustraciones a sus usuarios (Mosquera, 2017), por lo cual es importante desarrollar procedimientos de contingencia que permitan no detener el desarrollo de las actividades educativas programadas.

\section{CONCLUSIÓN}

La migración temporal a la educación virtual impulsó el uso de herramientas tecnológicas. Con ello, más allá de impulsar habilidades tecnológicas, se incentivó el desarrollo de nuevas competencias investigativas en los estudiantes, con lo cual se fomentó el autoaprendizaje y la disertación de los contenidos de sus materias. Sin embargo, se debe remarcar que incluso si la plataforma educativa es intuitiva, no se ha dejado de prescindir de la labor del docente. La metodología didáctica adaptada a la educación virtual requiere desarrollar aulas virtuales interactivas que fomente la participación autónoma de los estudiantes.

La adaptación al entorno virtualizado, tanto para el docente como para los estudiantes, es un requerimiento necesario dentro del proceso de migración de la 
educación hacia un entorno virtualizado. Los entornos virtuales interactivos permiten que la educación evolucione, rompiendo los paradigmas anteriores caracterizado por la pasividad del educando; en tal sentido la educación virtual está mostrando nuevos horizontes para el futuro de la educación.

En la actualidad, los docentes requieren contar con competencias concordantes con el progreso tecnológico de la educación. Es imprescindible que las instituciones educativas desarrollen esfuerzos permanentes en la formación tecnológica de los docentes. Se requiere que los docentes realicen un uso correcto de las herramientas tecnológicas disponibles en la institución, debido que luego serán los responsables de proporcionar e incentivar el uso de estas con los estudiantes.

Es imperativo que quienes ejercen la labor de docente, dispongan también de habilidades técnicas para hacer un uso eficaz e inteligente de las TIC. Lograr estas competencias en los docentes permitirá expandir el campo de acción de la educación; desde el entorno de un aula de clase hacia un campo colaborativo de nivel internacional; lo cual es posible gracias a las ventajas de accesibilidad que brinda la tecnología. Por lo tanto, es necesario que los docentes revaloren el uso de las TIC como herramientas que van a transformar la educación como la conocemos hoy.

El enfoque de la educación virtualizada exige que tanto docentes como estudiantes adquieran nuevas habilidades educativas, tanto para el desarrollo de contenidos como para su aplicación en el aula de clase. De esta forma, la educación virtualizada abre un campo novedoso para la educación, que cuenta con el potencial de expandir el aprendizaje y ampliar la interconexión de sus usuarios, coincidiendo con el desarrollo de una educación globalizada. 


\section{REFERENCIAS BIBLIOGRÁFICAS}

Andréu-Abela, J. (2001). Las técnicas de análisis de contenido: Una revisión actualizada. Centro de Estudios Andaluces. Disponible en: https://cutt.ly/ld3Vohu

Badia, A., Garcia, C., \& Meneses, J. (2017). Approaches to teaching online: Exploring factors influencing teachers in a fully online university. British Journal of Educational Technology, 48(6), 1193-1207. Disponible en: https://doi.org/10.1111/bjet.12475

Barba, J., Barba-Martín, R., \& González-Calvo, G. (2015). De la domesticación de la investigación cualitativa al reto de reinventarse. Revista Guillermo de Ockham, 13(1), 51. Disponible en: https://doi.org/10.21500/22563202.1687

Andréu-Abela, J. (2001). Las técnicas de análisis de contenido: Una revisión actualizada. Centro de Estudios Andaluces. Disponible en: https://cutt.ly/ld3Vohu

Badia, A., Garcia, C., \& Meneses, J. (2017). Approaches to teaching online: Exploring factors influencing teachers in a fully online university. British Journal of Educational Technology, 48(6), 1193-1207. Disponible en: https://doi.org/10.1111/bjet.12475

Barba, J., Barba-Martín, R., \& González-Calvo, G. (2015). De la domesticación de la investigación cualitativa al reto de reinventarse. Revista Guillermo de Ockham, 13(1), 51. Disponible en: https://doi.org/10.21500/22563202.1687

Borgobello, A., Sartori, M., \& Sanjurjo, L. (2019). Entornos virtuales de enseñanza y aprendizaje. Experiencias y expectativas de docentes universitarios de Rosario, Argentina. ESPACIOS EN BLANCO. Revista de Educación, 1(30), 41-58. Disponible en: https://doi.org/10.37177/unicen/eb30-263

Carmona-Suarez, E. J. (2017). Buenas prácticas en la educación superior virtual a partir de especificaciones de estándares e-Learning. Sophia, 13(1), 13-26. Disponible en:

https://doi.org/10.18634/sophiaj.13v.1i.345

Duarte-Herrera, M., Valdes Lozano, D. E., \& Montalvo Apolín, D. E. (2019). Estrategias disposicionales y aprendizajes significativos en el aula virtual. Revista Educación, 43(2), 30. Disponible en: https://doi.org/10.15517/revedu.v43i2.34038

Fidalgo-Blanco, Á., Sein-Echaluce, M. L., \& García-Peñalvo, F. J. (2015). Methodological approach and technological framework to break the current limitations of MOOC model. Journal of Universal Computer Science, 21(5), 712-734.

López-Catalán, L., \& Prieto-Jiménez, E. (2018). Tendencias innovadoras en la formación on-line. La oferta web de postgrados e-learning y blendedlearning en España. Pixel-Bit, Revista de Medios y Educación, 53, 93-107. Disponible en:

https://doi.org/10.12795/pixelbit.2018.i53.06

Medina-Gamero, A. (2019). La virtualidad de la educación, un reto en el aprendizaje universitario. Políticas, Tensiones y Tendencias de La Educación a Distancia y Virtual En América Latina 1, X(/iisue.20072872e.2019.29.532), 215-217. Disponible:

https://doi.org/https://doi.org/10.22201/iisue.20072872e.2019.29.532 
Mieles-Barrera, M. D., Tonon, G., \& Alvarado-Salgado, S. V. (2015). Investigación cualitativa: el análisis temático para el tratamiento de la información desde el enfoque de la fenomenología social. In Universitas Humanística (Vol. 13, Issue 1). Disponible en: https://cutt.ly/rd3VAML

Montes-Martínez, R., Prado-Salazar, J. R., \& Paz-Rebolloso, C. M. (2019). Alfabetización informacional y digital mediante b-learning. InterCambios. Dilemas y Transiciones de La Educación Superior, 6(1), 13-22. Disponible en: https://doi.org/10.29156/inter.6.1.1

Morado, M. F. (2017). El acompañamiento tecno-pedagógico como alternativa para la apropiación de tecnología en docentes universitarios. Actualidades Investigativas En Educación, 17 (3). Disponible en: https://doi.org/10.15517/aie.v17i3.29688

Morado, M. F., Ocampo Hernández, S., \& Ocampo, S. (2018). Una experiencia de acompañamiento tecno-pedagógico para la construcción de entornos virtuales de aprendizaje en educación superior. Revista Educación, 43 (1), 43-60. Disponible en: https://doi.org/10.15517/revedu.v43i1.28457

Mosquera, L. H. (2017). Impact of implementing a virtual learning environment (VLE) in the EFL classroom. Ikala, 22(3), 479-498. Disponible en: https://doi.org/10.17533/udea.ikala.v22n03a07

Rivera-Laylle, L. I., Fernández-Morales, K., Guzmán-Games, F. J., \& Eduardo-Pulido, J. (2017). La aceptación de las TIC por profesorado Universitario: Conocimiento, actitud y practicidad. Revista Electronica Educare, 21(3), 1-18. Disponible en: https://doi.org/10.15359/ree.21-3.6

Rodríguez Gómez, D., Castro Ceacero, D., \& Meneses, J. (2018). Usos problemáticos de las TIC entre jóvenes en su vida personal y escolar. Comunicar: Revista Científica Iberoamericana de Comunicación y Educación, 56, 91-100.

Sistema de información sobre tendencias educativas en América Latina-SITEAL. (2014). Políticas TIC en los sistemas educativos de América Latina. IIPE-UNESCO. Sede Regional Buenos Aires. Disponible en: http://archivo.siteal.iipe.unesco.org/sites/default/files/siteal_informe_ 2014_politicas_tic.pdf

Tapia-Repetto, G., Gutiérrez, C., \& Tremillo-Maldonado, O. (2019). Nuevas tecnologías en educación superior. Estudio de percepción en estudiantes acerca del uso de WhatsApp y Entornos Virtuales de Aprendizaje (Plataforma Moodle). Odontoestomatología, 21(33), 28-36. Disponible en: https://doi.org/10.22592/ode2019n33a5

Torres, C. E. T., \& Rodríguez, J. C. (2019). Los entornos de aprendizaje inmersivo y la enseñanza a cibergeneraciones. Educação e Pesquisa, 45, 1-20. Disponible en: https://doi.org/10.1590/s16784634201945187369

Tünnermann, C. (2008). Modelos educativos y académicos. In E. Hispamer (Ed.), Editorial Hispamer (Primera ed).

Veytia-Bucheli, M. G., \& Contreras-Cipriano, Y. (2019). Factores motivacionales para la investigación y los objetos virtuales de aprendizaje en estudiantes de maestría en Ciencias de la Educación. RIDE Revista Iberoamericana Para La Investigación y El Desarrollo Educativo, 9(18), 84-101. Disponible en: https://doi.org/10.23913/ride.v9i18.413 